\title{
Rich Approach for Semantic Segmentation using Neuro-Fuzzy Technique
}

\author{
Kanwaljit Kaur $^{1}$, Gurjit Singh ${ }^{2}$ \\ M. Tech Scholar, Department of E.C.E, ACET, ASR, Punjab ${ }^{1}$ \\ Assistant Professor, Department of E.C.E, ACET, ASR, Punjab ${ }^{2}$
}

\begin{abstract}
Image segmentation is the process of partitioning an image into multiple segments, so as to change the representation of an image into something that is more meaningful and easier to analyse. Semantic segmentation makes an effort to partition the image into semantically meaning parts also to classify each part into one among predetermined categories. Semantic segmentation may be a classification task. Semantic segmentation is that the task of cluster components of pictures along that belongs to a similar object category.
\end{abstract}

Keywords: 3D Entangled, Semantic Segmentation, Neuro-Fuzzy Network

\section{INTRODUCTION}

Image processing is the general issue in today's era, when we work with computer vision. It is in itself, a broad view to be considered. In order to process the image, we need to segment it so that it would become easier for the computer to understand Image segmentation is the process of segmenting the image into various segments that could be used for the further applications such as: Image understanding model, Robotics, Image analysis, Medical diagnosis, etc.[1] Image segmentation is the process of partitioning an image into multiple segments, so as to change the representation of an image into something that is more meaningful and easier to analyse. Segmentation technique, basically convert the complex image into the simple image as shown in the figure 1.

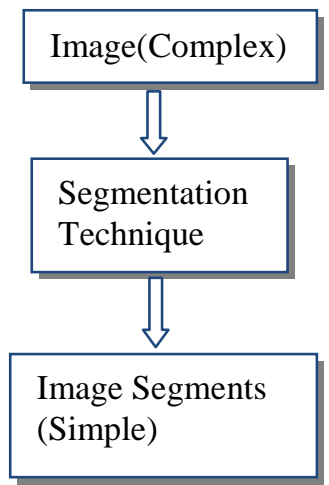

Figure 1.Segmentation technique [1]

Segmentation is a division of a image into many logical parts but segmentation clearly define the meaning of these parts. On the opposite hand semantic segmentation makes an effort to partition the image into semantically meaning parts also to classify each part into one among pre- determined categories. Semantic segmentation may be a classification task. Semantic segmentation is that the task of cluster components of pictures along that belongs to a similar object category.

\section{LITERATURE REVIEW}

3D All the Way: Semantic Segmentation of Urban Scenes from Start to End in 3D [3]

This paper proposed a new approach for semantic segmentation of 3D city models. Starting from a SfM reconstruction of a street-side scene, we perform classification and façade splitting purely in 3D, obviating the need for slow image based semantic segmentation methods. This shows that a properly trained pure-3D approach produces high quality labeling, with significant speed benefits (20x faster) allowing us to analyze entire streets in a matter of minutes. Additionally if speed is not of the essence, the 3D labeling can be combined with the results of a state-of-the-art 2D classifier further boosting the performance. Further, a novel facade separation based on semantic nuances between 
facades. Finally, inspired by the use of architectural principles for 2D facade labeling new , 3D-specific principles and an efficient optimization scheme based on an integer quadratic programming formulation.

Global localization of 3D anatomical structures by pre-filtered Hough Forests and discrete optimization [4]

The accurate localization of anatomical landmarks is a challenging task, often solved by domain specific approaches. Author proposed a method for the automatic localization of landmarks in complex, repetitive anatomical structures. The key idea is to combine three steps: (1) a classifier for pre-filtering anatomical landmark positions that (2) are refined through a Hough regression model, together with (3) a parts-based model of the global landmark topology to select the final landmark positions. During training landmarks are annotated in a set of example volumes. A classifier learns local landmark appearance, and Hough repressors' are trained to aggregate neighborhood information to a precise landmark coordinate position. A non-parametric geometric model encodes the spatial relationships between the landmarks and derives a topology which connects mutually predictive landmarks. During the global search they classify all voxels in the query volume, and perform regression-based agglomeration of landmark probabilities to highly accurate and specific candidate points at potential landmark locations. They encode the candidates' weights together with the conformity of the connecting edges to the learnt geometric model in a Markov Random Field (MRF). By solving the corresponding discrete optimization problem, the most probable location for each model landmark is found in the query volume. They show that this approach is able to consistently localize the model landmarks despite the complex and repetitive character of the anatomical structures on three challenging data sets (hand radiographs, hand CTs, and whole body CTs), with a median localization error of $0.80 \mathrm{~mm}, 1.19 \mathrm{~mm}$ and $2.71 \mathrm{~mm}$, respectively.

Semantic Segmentation as Image Representation for Scene Recognition [5]

They introduce a novel approach towards scene recognition using semantic segmentation maps as image representation. Given a set of images and a list of possible categories for each image, our goal is to assign a category from that list to each image. Their approach is based on representing an image by its semantic segmentation map, which is a mapping from each pixel to a pre-defined set of labels. Among similar high-level approaches, has the capability of not only representing what semantic labels the scene contains, but also their shapes, sizes and locations. They obtain state-ofthe-art results over Sift flow and Msrc datasets.

\section{A Study Analysis on the Different Image Segmentation Techniques [1]}

Image segmentation is an important image processing step, and it is used everywhere if you want to analyze what is inside the image. Image segmentation basically provides the meaningful objects of the image. This paper represents the various image segmentation techniques that could be used in the segmentation algorithm. Whenever they work with the image in any application, initial step is to segment the image in order to solve its complexity. The segmentation of images is the basic thing for understanding the images. It is used in the Image processing applications, Computer vision etc. In this paper, two categories are emphasized: Edge based and region based segmentation, which further includes their respective techniques.

\section{PROPOSED METHODOLOGY}

In our proposed methodology minimum and maximum strength of systematic sets are denoted for chosen row and column intensity values for reducing the storage space. For reducing storage space the interim sequence is also stored. On the basis of Intensity value intervals the Neuro-fuzzy network procedure are formed for document image for favorable intensity various interval set are formed. The shape and intensity of pixel from various intervals helps in defining the feature of image. The Neuro-fuzzy network work on defining various desired intensity layers for retrieving desired results from interval of image portion. If the pixel with uniform intensity arises they are considered as similar region .the proposed technique Neuro-Fuzzy Network help in identifying this similar region for better selection of region for efficient results.

\section{ALOGRITM: Neuro- Fuzzy Network}

Step 1: Scan the document image store it in the database

Step 2: Using interval generates intensity values for the image.

Step3: Select different interval for specific intensity value

Step4: Apply Neuro- fuzzy procedure and store the value for favorable portion intensity value

Step5: finds the result for various intervals

Step 6: Record the intensity values

Step 7: Store the final results

Step 8: For each training image repeat process

Step 9: The segmented image Displayed

Step10: End 


\section{RESULTS AND ANALYSIS}

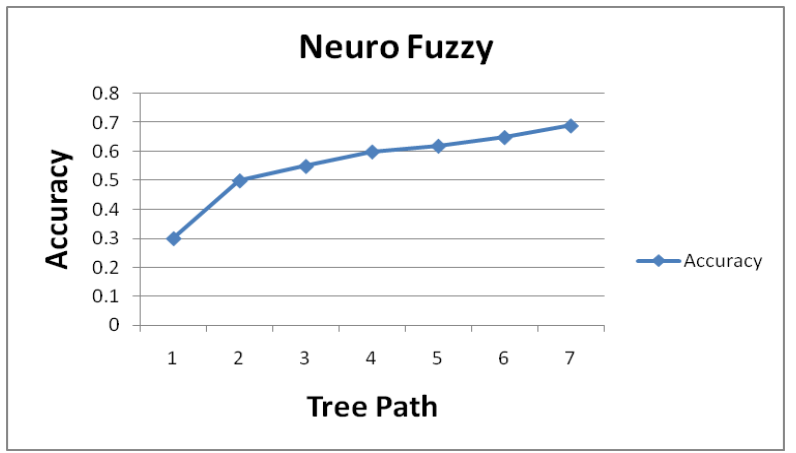

Figure2. The above figure shows the results with proposed technique NF.

The graph shows increase in accuracy value with increase in tree path

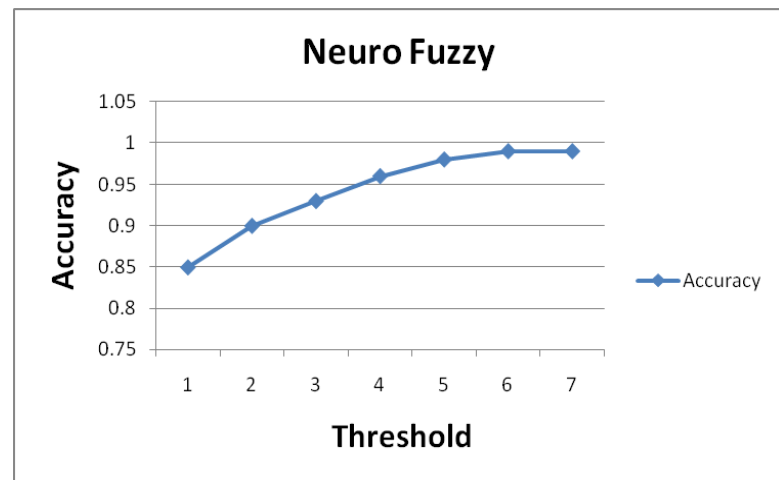

Figure 3. The above figure shows the results with proposed technique NF.

The graph shows increase in accuracy value with increase in threshold

\section{CONCLUSION}

In this work the problem of bottom-up figure-ground segmentation, both as an image Segmentation task, and as a perceptual grouping problem. We presented a comprehensive overview of current research in both fields, and discussed reasons why despite a vast research effort, image segmentation and perceptual grouping on unconstrained images continue to be extremely challenging. The Neuro-Fuzzy network technique is proposed for efficient semantic segmentation. The proposed technique is capable of finding relation between the objects, structure and indoors. The proposed technique is evaluated on the basis of intervals intensity value for favourable portion for desired results .the proposed technique provide better results as compared to previous technique.

\section{REFERENCES}

[1]. Sujata Saini and Komal Arora “A Study Analysis on the Different Image Segmentation Techniques” International Journal of Information \& Computation Technology, ISSN 0974-2239 Volume 4, Number 14 (2014).

[2]. Daniel Wolf, Johann Prankl, and Markus Vincze "Enhancing Semantic Segmentation for Robotics: The Power of 3-D Entangled Forests" IEEE ROBOTICS AND AUTOMATION LETTERS, VOL. 1, NO. 1, JANUARY 2016.

[3]. Andelo Martinovic, Jan Knopp, Hayko Riemens chneider and Luc Van Gool "3D All The Way: Semantic Segmentation of Urban Scenes From Start to End in 3D" in CVPR 2015.

[4]. René DonnerBjoern H. MenzeHorst BischofGeorg Langs Global localization of 3D anatomical structures by pre-filtered filtered hough forests and discrete optimization 2013

[5]. Ahmed Bassiouny, Motaz El-Saban: Semantic segmentation as image representation for scene recognition

[6]. A. Garcia-Garcia, S. Orts-Escolano, S.O. Oprea, V. Villena-Martinez, and J. Garcia-Rodriguez "A Review on Deep Learning Techniques Applied to Semantic Segmentation" arXiv:1704.06857v1 [cs.CV] 22 Apr 2017

[7]. Mahmud S. Alkoffash and Mohammed J. Bawaneh "A Survey of Digital Image Processing Techniques in Character Recognition" IJCSNS VOL.14 No.3, March 2014

[8]. Ashraf A. Aly1, Safaai Bin Deris2, Nazar Zaki3 "Research review for digital image segmentation techniques" International Journal of Computer Science \& Information Technology (IJCSIT) Vol. 3, No 5, Oct 2011

[9]. Thanos Athanasiadis, PhivosMylonas, YannisAvrithis and StefanosKollias "Semantic Image Segmentation and Object Labelling " IEEE transactions on circuits and systems for video technology, vol. 17, no. 3, march 2007

[10]. Pablo Arbel'aez,Bharath Hariharan,ChunhuiGu, Saurabh Gupta, Lubomir Bourdev "Semantic Segmentation using Regions and Parts" In Proc. CVPR, 2012 